\title{
Evolution of CDM halos and the Milky Way satellites
}

\author{
Jürg Diemand \\ Department of Astronomy and Astrophysics, University of California, \\ 1156 High Street, Santa Cruz, CA 95064, USA \\ email: diemand@ucolick.org
}

\begin{abstract}
A cold dark matter halo big enough to host the Milky Way contains hundreds of subhalos massive enough to host dwarf galaxies. The difference to the much smaller observed number of satellite galaxies seems to be a problem for CDM. The galaxy number density profile and disk like configuration are also different form the total subhalo populations in CDM simulations. A number of different models of dwarf galaxy formation which are able to reproduce the right number of luminous subhalos have been proposed. Some of them also give the right radial distributions and make disk like configurations more probable. Additional information about the typical formation times and sites of dwarf galaxies can be found in the stellar halo of the Milky Way, i.e. from the stellar debris of tidally disrupted dwarfs. A stellar halo with a realistic concentration is obtained when most dwarfs form early (before redshift 10) in small halos (virial mass above $10^{8}$ solar masses). This mass scale found from simulations of dark matter structure formation coincides with the virial temperature of $10^{4} \mathrm{~K}$ which is needed for efficient atomic cooling.
\end{abstract}

Keywords. methods: n-body simulations,methods: numerical, Galaxy: halo galaxies: dwarf, galaxies: evolution, galaxies: formation, dark matter

\section{Introduction}

In a $\Lambda$ CDM universe $\left(0.3,0.7, \sigma_{8}=0.9\right)$ a dark matter halo big enough to host the Milky Way contains $300 \pm 100$ subhalos with circular velocities above $10 \mathrm{~km} / \mathrm{s}$ (Diemand, Moore \& Stadel 2004b). These subhalos would all be massive enough to contain a dwarf galaxy but there are only about 10 observed satellites around our Galaxy. This discrepancy, the so called 'missing satellites problem' (Moore et al. 1999; Klypin et al. 1999), is one of the challenges faced by $\Lambda$ CDM. Other open issues also concern the internal halo structure predicted by CDM: the steep density profiles (Moore 1994; Flores \& Primack 1994) and that the distribution of angular momentum is different form the one observed in disk galaxies (e.g. Primack 2003). There are two different ways of dealing with the missing satellites problem, the first one is to change structure formation in a way which dramatically reduces the number of subhalos (e.g. warm dark matter). Here we consider a second class of solutions: The CDM subhalo predictions are not altered but galaxy formation somehow does not take place in all subhalos and leaves most of them dark.

\section{Do only the most massive subhalos host dwarfs?}

Stoehr et al. (2002) suggested that the stellar velocity dispersions of dwarfs are much smaller than the maximum of the circular velocity of the subhalo they live in and that the most massive subhalos correspond to the observed satellites. Small stellar dispersions in the inner part of massive subhalos are possible if the subhalo density profile is shallow or even flat in the inner part. Stoehr et al. (2002) suggested a subhalo profile which is much 
shallower than the NFW-like profiles typically found in field halos. However Kazantzidis et al. (2004) found that subhalo density profiles in high resolution cosmological simulations resemble those of field halos over the entire resolved range. While mass is removed at all radii, tides are more effective further out and the density of dark matter bound to a subhalos is lowered much more in the outer part. The inner profile slopes are unchanged after the tidal stripping (Kazantzidis et al. 2004). Therefore we expect similar inner profiles for subhalos and field halos. In field halos highest resolution cosmological simulations now start to resolve an inner cusp, i.e. a region with constant logarithmic slope, for an average cluster halo an asymptotic slope of -1.2 is found (Diemand et al. 2005a).

One has to assume an anisotropy profile $\beta(r)$ for the stars to convert the subhalo density profile into a stellar line of sight velocity dispersion profile with the Jeans equation. For the $\beta(r)$ which give realistic velocity dispersion profiles one finds $v_{c}$ values which are not much higher than the observed dispersions. For example for the subhalo around Fornax one finds a $v_{c}$ of $20-30 \mathrm{~km} / \mathrm{s}$ (Kazantzidis et al. 2004) which is lower than the $v_{c}$ values of the ten most massive subhalos.

The radial distribution of the most massive subhalos at $\mathrm{z}=0$ is more extended than the dark matter halo (Ghigna et al. 1998; Ghigna et al. 2000; De Lucia et al. 2004; Diemand, Moore \& Stadel 2004b). The number density of the observed satellites follows roughly the dark halo density, i.e. the most massive subhalos are too far out to host our dwarf galaxies (Moore 2001; Taylor, Silk \& Babul 2003).

\section{Only early forming subhalos host dwarfs}

Due to tidal stripping the present bound mass of subhalos can be much smaller than the mass before accretion. Dwarf galaxies probably have formed before accretion therefore a selection of luminous subhalos should rather depend on their mass (or $v_{c}$ or virial temperature) at some time before accretion. Unlike selections by bound mass at $\mathrm{z}=0$ selections before accretion give the right radial extent of luminous subhalos (Moore 2001; Nagai \& Kravtsov 2005; Moore et al. 2005).

There are several plausible physical processes which may suppress gas accretion and star formation in small dark matter halos. One possibility is that small halos accrete gas efficiently only before reionisation. In this case only the earliest forming small halos from stars. It is possible to get the right number of surviving luminous subhalos for a number of plausible reionisation redshifts $\left(z_{R E}=5-12\right)$ and star formation efficiencies (Bullock, Kravtsov \& Weinberg 2000). After reionisation the increasing UV background heats up the intergalactic medium and the minimal halo mass needed for efficient gas accretion grows with time, i.e. there is a filtering mass scale $\mathrm{M}(\mathrm{z})$ which decides if a halo is massive enough to become luminous (e.g. Gnedin 2000). Such a filtering mass function was implemented to populate the subhalos of high resolution cosmological dark matter simulations by Kravtsov, Gnedin \& Klypin (2004; hereafter KGK04). For each surviving subhalo the simulations were used to extract the detailed histories of mass accretion, mass loss due to tidal stripping, tidal forces and orbits. In this model most luminous subhalos were much more massive before accretion, about 90 percent had a virial mass larger than $10^{10} M_{\odot}$ and $v_{c}>40 \mathrm{~km} / \mathrm{s}$. Tidal stripping reduces both the bound mass and the maximal circular velocity since the density is lowered at all radii (Kazantzidis et al. 2004). This point was neglected in semi-analytic models like the one by Bullock, Kravtsov \& Weinberg (2000) and it is essential to get the correct velocity function in the model by KGK04.

The smallest luminous halos $\left(v_{c}<30 \mathrm{~km} / \mathrm{s}\right)$ could loose their gas due to photoevaporation after reionisation or also due to supernova feedback (e.g. Dekel, Lin, these 


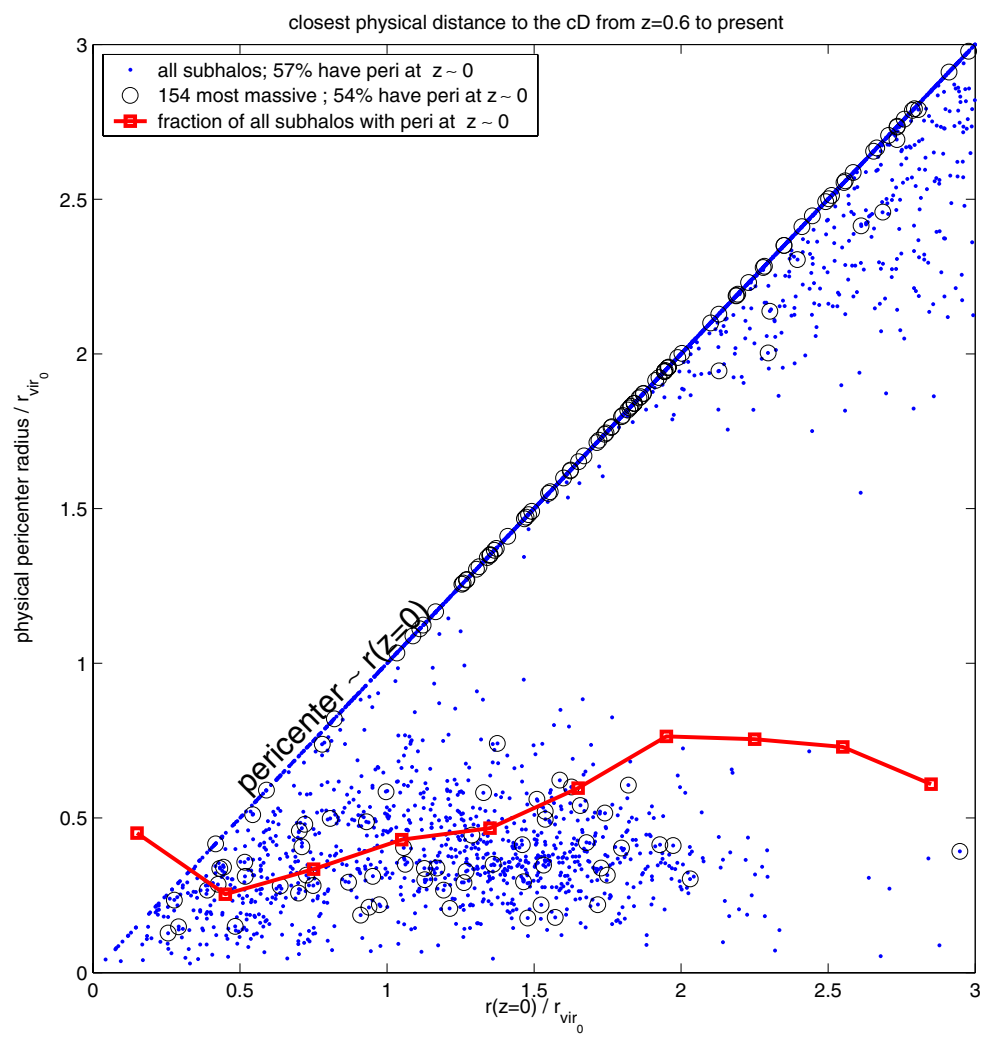

Figure 1. Closest physical distance to the halo center form $\mathrm{z}=0.6$ to present vs. distance form the halo center today. The red line gives the fraction of (sub)halos that have their peri-center today (within the time resolution of 0.6 Gyrs). The points in the lower right corner show that many halos well outside the primary halo today passed deep through it earlier.

proceedings). The smaller dwarfs have only old stellar populations but the larger satellite galaxies manage to hold on to their gas and they from stars also at low redshift, maybe in bursts triggered by tidal forces (Mayer et al. 2001). Tidal forces can also change the morphologies of galaxies (Moore et al. 1996a; Mastropietro et al. 2005), in the Local Group they transform dwarf irregulars to dwarf spheroidals (Mayer et al. 2001) explaining the morphological segregation. Exceptions (dwarf spheroidals far away form their host like Cetus and Tucana) are allowed since the entire history of all tidal forces endured determines todays appearance. Some halos beyond 2 virial radii at $\mathrm{z}=0$ have been closer than half a virial radius at some earlier time (see Figure 1 and Moore, Diemand \& Stadel 2004), such halos could host remote dwarf spheroidals like Cetus and Tucana.

\section{Destroyed dwarfs build up the stellar halo}

Most dwarf galaxies which ever existed in the Local Group were destroyed by the tidal forces of the MW and M31 (or their main progenitors). Today their stars and globular cluster make up the halos of the MW and M31. The surviving number, velocity function and radial distribution of dwarfs can be explained with a time dependent filtering mass $\mathrm{M}(\mathrm{z})$ which rises after the reionisation time $z_{R E}$. Such models work for a wide range of plausible $z_{R E}$ values (Bullock, Kravtsov \& Weinberg 2000; KGK04). In this section we 
examine the additional constraints form the spatial distribution of the disrupted dwarfs, i.e. the stellar halo. For simplicity we assume a step function for $\mathrm{M}(\mathrm{z})$ : halos have to reach some minimal mass $M_{\min }$ at the reionisation redshift $z_{R E}$ in order to accrete gas and form stars. For a large number of $\left(M_{\min }, z_{R E}\right)$ pairs we identify these early star forming proto-galaxies in a cosmological simulation and trace them to $z=0$. Then we analyze both the component bound inside surviving subhalos and the disrupted stellar halo component (see Figure 2).

Marking halos above some $M_{\min }$ at some $z_{R E}$ in four galaxy halos (each resolved with a few million particles in a cosmological simulation) as luminous we get realistic satellite populations for the $\left(M_{\min }, z_{R E}\right)$ pairs connected with the dashed line in Figure $3 \dagger$. Also plotted are the numbers of sigmas a peak of some mass collapsing at this redshift corresponds toł. The concentration of the stellar halo depends only on how rare (above how many sigmas) the small high redshift building blocks are, the rare ones form more biased towards the center and give colder, more concentrated and more radially anisotropic $\mathrm{z}=0$ distributions (Diemand, Madau \& Moore 2005b) 1 . To match the number density profile of the stellar halo and the metal poor globular clusters of the Galaxy its building blocks must form in collapsed fluctuations above $2.5 \sigma$ (Moore et al. 2005). This constraint intersects with the dashed line for producing a realistic satellite galaxy population at $M=10^{8} M_{\odot}$ and $z_{R E}=12$. This mass-redshift pair inferred from structure formation arguments alone corresponds an important physical scale for neutral gas: Such halos have viral temperatures just above $10^{4} K$ (dotted line), i.e. the infalling gas gets hot enough to allow efficient atomic line cooling.

In our scenario the filtering mass function follows the $10^{4} \mathrm{~K}$ line up to $z_{R E}=12$ and then rises steeply along the $2.5 \sigma$ line (or steeper) up to a few times $10^{10} M_{\odot}$ where the UV background cannot suppress gas accretion anymore. There are a few hundred luminous building blocks above $M=10^{8} M_{\odot}$ at $z_{R E}=12$ per galaxy halo, these are relatively rare peaks $(\geqslant 2.5 \sigma)$ and their formation sites are strongly biased towards the center of the galaxy forming region. Most of them merge together quite rapidly and about $95 \%$ of them are completely disrupted in this process. The outermost building blocks (see upper panel of Figure 2) fall in later and with larger orbital energy, they have good chances to survive the tidal forces and to become one of the satellite galaxies at $\mathrm{z}=0$.

\section{Is the disk like configuration a serious problem for CDM?}

The satellite galaxies around the Milky Way lie close to a plane and the probability to draw such a configuration from a spherical distribution is practically zero (Kroupa, Theis \& Boily 2005). But CDM halos are triaxial and the luminous subhalos in the KGK04 scenario trace the underlying mass distribution (Zentner et al. 2005). Also the small number of observed satellites and the fact that most of them are nearby makes it easier to find a plane like configuration like the observed one (Kang et al. 2005; Zentner et al. 2005). The Milky Way resembles their modeled CDM galaxy-satellite systems in about $10 \%$ of the cases, which is still a remarkably low value but not low enough to indicate a serious problem for CDM.

$\dagger$ After $\mathrm{z}=12$ this line is similar to the filtering mass function for $z_{R E}=10$ model by KGK04.

$\ddagger$ Using the dashed line as filtering function includes some rare, early peaks, but the lowest $\sigma$ allowed will dominate. That is why in the KGK04 model most luminous subhalos are above $10^{10} M_{\odot}$ before accretion.

व At lower redshifts $(z<3)$ the relevant building blocks are more massive $M \simeq 10^{10} M_{\odot}$ and the $\mathrm{z}=0$ distributions start do depend also on which parts of them are marked and traced to $\mathrm{z}=0$. 


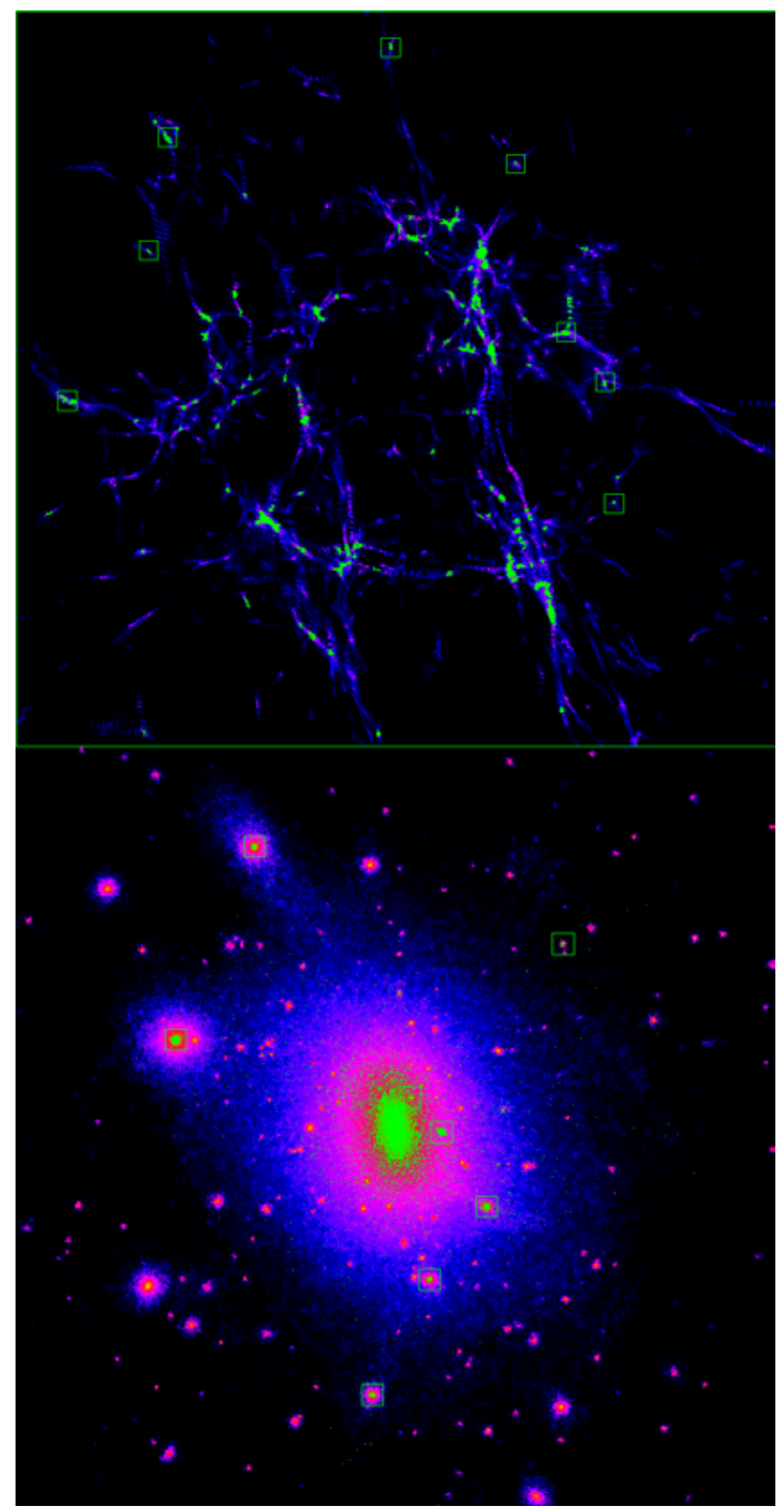

Figure 2. The upper panel shows the dark matter density distribution at a redshift $z=12$ from a region that will form a single galaxy at $z=0$ (lower panel) in a cosmological simulation. The green regions show the halos with mass above $10^{8} M_{\odot}$ at $z=12$. The same marked particles are plotted green also at $z=0$. Most of the rare peaks are located near the center of the galaxy today. The squares in both panels indicate those first objects that survive as satellite galaxies today orbiting within the final galactic mass halo. Most proto-galaxies were destroyed and have built up a concentrated stellar halo by $\mathrm{z}=0$. 


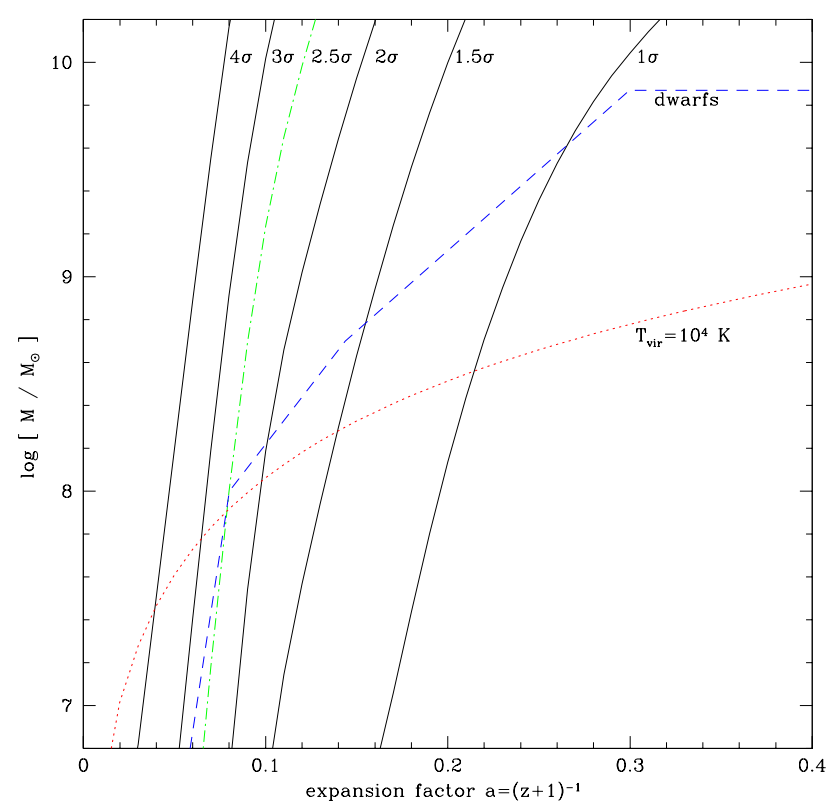

Figure 3. Mass-redshift diagram for different formation sites and times of proto-galaxies. Proto-galaxies are assumed to form in all halos above $M$ at redshift $\mathrm{z}$, their stars are then traced to $\mathrm{z}=0$. Using the $(\mathrm{M}, \mathrm{z})$ pairs given by the dashed line produces about ten surviving luminous subhalos per galaxy. The solid lines give the number of sigmas a peak of mass $M$ collapsing at redshift $\mathrm{z}$ corresponds to. The concentration of the disrupted material depends only on which sigma line dominates the selected population. To reach the concentration of the stellar halo of the Galaxy peaks below $2.5 \sigma$ must be excluded since low $\sigma$ material has a too extended $\mathrm{z}=0$ distribution. Halos with a virial temperature of $10^{4} K$ lie on the dotted line.

If the dwarfs are descendants of early high sigma peaks (previous section and Moore et al. 2005) the shape of the stellar halos and of the dwarf galaxy configuration is expected to be substantially more elongated than the total dark halo (Diemand, Madau \& Moore 2005b). Figure 4 shows the $\mathrm{z}=0$ axis ratios of high sigma material and the total halo averaged over four galaxy size CDM halos form a cosmological simulation. Perhaps surprising the oldest components are the least round, one could have naively expected that they would have mixed and relaxed into a similar or rounder shape as the entire dark halo. The shapes of stellar halo, metal poor globular cluster and dwarf halo configuration are strongly correlated in this scenario and are about $\mathrm{c} / \mathrm{a}=0.4$ and $\mathrm{b} / \mathrm{a}=0.5$ on average with a halo to halo scatter of about 0.1 . Their orbits become more radially anisotropic with radius: $\beta(r)=1-0.5 \sigma_{t}^{2} / \sigma_{r}^{2}$ grows from zero near the galactic center to $0.45 \pm 0.05$ at our position and to about $0.6 \pm 0.25$ near the virial radius.

The configuration of satellites with respect to the disk planes of their hosts (Kroupa, Theis \& Boily 2005; Holmberg 1969; Sales \& Lambas 2004) certainly deserves further studies both observational and theoretical, but probably large samples of well observed and well simulated systems have to be used for detailed comparisons since the correlation of baryonic angular momentum (disk normal axis) to halo shape (satellite configuration) are only weak (van den Bosch et al. 2002, Faltenbacher et al. 2002). 

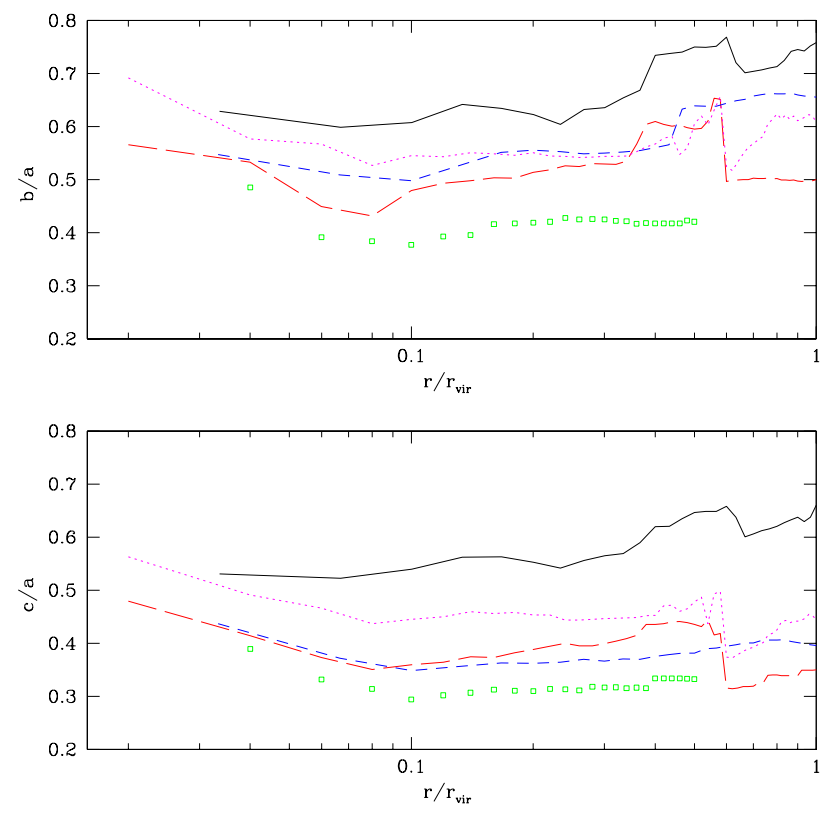

Figure 4. Shape profiles of high sigma material at $z=0$ averaged over four galaxy halos. The shapes are plotted for 3.5 sigma material (groups with virial mass above $4.9 \times 10^{7} M_{\odot}$ at $\mathrm{z}=13.7$, squares), 3 sigma material $\left(\geqslant 5.9 \times 10^{6} M_{\odot}\right.$ at $\mathrm{z}=13.7$ long dashed and groups above $2.2 \times 10^{8} M_{\odot}$ at $\mathrm{z}=10.5$ short dashed) and 2 sigma material $\left(\geqslant 4.8 \times 10^{7} M_{\odot}\right.$ at $\mathrm{z}=7.5$, dotted lines $)$. The shape of the entire dark matter halos are plotted with solid lines.

\section{Summary}

Missing satellites must not be a problem for CDM: They can be regarded as evidence for when and on what halo mass scales galaxy formation is suppressed. Models which include such a suppression in halos below a minimum mass at some time before accretion can produce the right number and velocity function of satellite galaxies and the radial distribution comes out right too. The disk like configuration of dwarfs around the Milky Way is not the most common outcome in such models, but it is not rare enough to disprove them either.

\section{Acknowledgements}

This contribution is in part based on work done in collaboration with Ben Moore, Joachim Stadel and Piero Madau. I would like to thanks the Swiss National Science Foundation and the IAU for financial support.

\section{References}

Bullock, J.S., Kravtsov, A.V. \& Weinberg, D.H. 2000, ApJ 539, 517

De Lucia G., Kauffmann G., Springel V., White, S.D.M., Lanzoni, B., Stoehr, F., Tormen, G. \& Yoshida, N. 2004, MNRAS, 348, 333

Diemand, J., Moore, B. \& Stadel, J. 2004b, MNRAS 352, 535

Diemand, J., Zemp, M., Moore, B., Stadel, J. \& Carollo, M. 2005a, submitted, astro-ph/0504215

Diemand, J., Madau, P. \& Moore B., "The distribution and kinematics of high sigma peaks in CDM halos", 2005b, in prep.

Faltenbacher, A., Kerscher, M., Gottloeber, S. \& Mueller, V. 2002 A\&A 395, 1 
Flores, R.A. \& Primack, J.R. 1994, ApJ 427, L1

Ghigna, S., Moore, B., Governato, F., Lake, G., Quinn, T. \& Stadel J. 1998, MNRAS 300, 146

Ghigna, S., Moore, B., Governato, F., Lake, G., Quinn, T. \& Stadel, J. 2000, ApJ 544, 616

Gnedin, N.Y. 2000, ApJ 542, 535

Holmberg, E. 1969, Arkiv. Astr 5, 305

Kang, X., Mao, S., Gao, L. \& Jing, Y.P. 2005, A\& $A$ in press, arXiv:astro-ph/0501333.

Kazantzidis, S., Mayer, L., Mastropietro, C., Diemand, J., Stadel, J. \& Moore, B. 2004, ApJ 608,663

Klypin, A., Kravtsov, A.V., Valenzuela, O. \& Prada, F. 1999, ApJ 522, 82

Kravtsov, A.V., Gnedin, O.Y. \& Klypin, A.A. 2004, ApJ 609, 482

Kroupa, P., Theis, C. \& Boily, C.M. 2005, A\& $A$ 431, 517

Mayer, L., Governato, F., Colpi, M., Moore, B., Quinn, T., Wadsley, J., Stadel, J. \& Lake, G. 2001, ApJ 547, L123

Mastropietro, C., Moore, B., Mayer, L., Debattista, V.P., Piffaretti, R. \& Stadel J. 2005, MNRAS submitted, arXiv:astro-ph/0411648

Moore, B. 1994, Nature 370, 629

Moore, B., Katz, N., Lake, G., Dressler, A. \& Oemler, A.J. 1996, Nature 379, 613

Moore, B., Katz, N. \& Lake, G. 1996b, ApJ 457, 455

Moore, B., Ghigna, S., Governato, F., Lake, G., Quinn, T., Stadel, J. \& Tozzi, P. 1999, ApJ $524, \mathrm{~L} 19$

Moore, B., 2001, AIP Conf. Proc. 586, 20th Texas Symposium on relativistic astrophysics, 73

Moore, B., Diemand, J. \& Stadel, J. 2004, IAU Symposium 222 513

Moore, B., Diemand, J., Madau, P., Zemp, M. \& Stadel, J. "The assembly of the galactic halo", 2005 , in prep.

Nagai, D. \& Kravtsov A.V. 2005, ApJ 618, 557

Primack, J.R. 2003, IAUS 220 arXiv:astro-ph/0312549.

Sales, L. \& Lambas, D.G. 2004, MNRAS 348, 1236

Stoehr, F., White, S.D., Tormen, G. \& Springel, V. 2002, MNRAS 335, L84

Taylor, J.E., Silk, J. \& Babul, A. 2003, IAUS 220 astro-ph/0312086

van den Bosch, F.C., Abel, T., Croft, R.A.C., Hernquist, L. \& White, S.D.M. 2002, ApJ 576, 21

Zentner, R.A., Kravtsov, A.V., Gnedin, O.Y. \& Klypin A.A. 2005, ApJ in press, arXiv:astro$\mathrm{ph} / 0502496$.

\section{Discussion}

FERGuson: Does this model predict any trends of dwarf-galaxy properties with giantgalaxy mass?

DiEmAnD: Selecting luminous halos at the same $\mathrm{z}$ and $\mathrm{M}$, one gets similar mass fractions, distributions, and kinematics for the total old luminous component (bulge, halo, and dwarfs) in larger halos $\left(M_{v i r} \geqslant 10^{12} M_{\text {sun }}\right)$. The number of dwarfs would rise proportional to the halo mass, but I still have to check whether the same fraction of early halos survives in these larger halos. Most smaller systems $\left(M_{\text {vir }} \approx 10^{11} M_{\text {sun }}\right)$ have no stellar halo and dwarfs in this scenario.

RicotTi: In order to reproduce the radial distribution of galactic satellites you need to select $3 \sigma$ peaks. You decided to assume $M_{D M}>10^{8} M_{\text {sun }}$ and you found that these objects must form at $z<14$. If you do allow star formation in $M_{D M} \approx 10^{7} M_{\text {sun }}$ halos, at which redshift do you need to populate your dark halos?

DiEmAND: A halo of this mass corresponds to a $3 \sigma$ peak at about $z=16$ in this cosmology $\left(0.3,0.7, \sigma_{8}=0.9\right)$. 
MASHCHENKO: This is about your statement that low stellar velocity dispersion in dSphs implies that these galaxies cannot be hosted by most massive subhalos in the Milky Way halo. This is not correct, as in the case of stars having formed from cold gas with low angular momentum the stellar velocity dispersion can be much lower than that of DM.

DiEmAnD: My statement is correct for the observed Local Group dwarfs: Using the observed light profile, $\sigma$-profile, a cuspy halo, and assuming any reasonable anisotropy, one gets a circular velocity for the halo of Draco of about $30 \mathrm{~km} / \mathrm{s}$ (Kazantzidis et al. 2004), close to the observed $\sigma$.

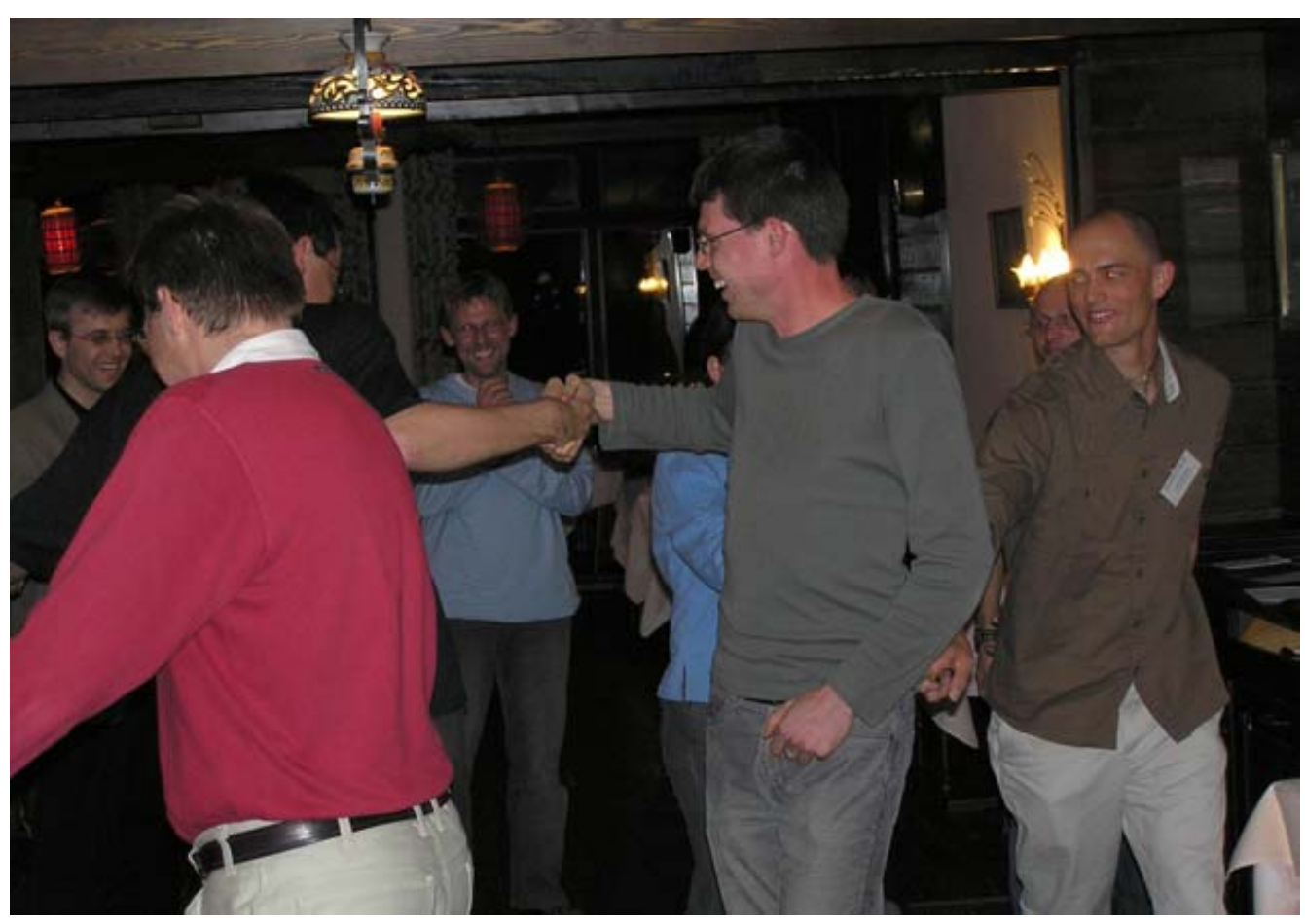

